\title{
Genomic mapping of pathways in endometrial adenocarcinoma and a gastrointestinal stromal tumor located in Meckel's diverticulum
}

\author{
MONIKA ENGLERT-GOLON ${ }^{1 *}$, BARTLOMIEJ BUDNY ${ }^{2 *}$, BARTOSZ BURCHARDT $^{1}$, ELZBIETA WROTKOWSKA ${ }^{2}$, \\ KATARZYNA ZIEMNICKA ${ }^{2}$, MAREK RUCHAŁA ${ }^{2}$ and STEFAN SAJDAK ${ }^{1}$
}

${ }^{1}$ Surgical Gynecology Clinic of the Gynecological and Obstetrics Clinical Hospital; ${ }^{2}$ Department of Endocrinology, Metabolism and Internal Diseases, Poznan University of Medical Sciences, 60-535 Poznan, Poland

Received November 16, 2014; Accepted October 13, 2015

DOI: $10.3892 / 01.2015 .4004$

\begin{abstract}
The present study reports the case of a 71-year-old female patient diagnosed with endometrial adenocarcinoma, which was confirmed by histopathology. In the course of performing an elective hysterectomy with adnexa removal, a solid tumor located in Meckel's diverticulum (MD) was identified and excised. Due to the unique nature of the lesion, the tumor tissue underwent broad mapping of any genomic alterations once the histopathological examination was completed. The genetic testing was conducted using a high-resolution microarray and resulted in the identification of 45 genomic abnormalities, including 4 chromosomal aneuploidies. Within those regions, alterations of 87 known cancer genes were assigned. The involvement of v-kit Hardy-Zuckerman 4 feline sarcoma viral oncogene homolog gene alteration was noted to be a key player for triggering gastrointestinal stromal tumor transformation for this unusual case. A total of 12 genes, showing mutual interaction in different cancer types or involved in diverse cellular processes, were identified. These reported data may shed light on the carcinogenesis of a rare MD tumor.
\end{abstract}

\section{Introduction}

Gastrointestinal stromal tumors (GISTs) are the most frequent mesenchymomas of the gastrointestinal tract with a smooth muscle origin. The application of immunohistochemistry to the study of GISTs, provides novel insights into the disorder,

Correspondence to: Professor Stefan Sajdak, Surgical Gynecology Clinic of the Gynecological and Obstetrics Clinical Hospital, Poznan University of Medical Sciences, Polna 33, 60-535 Poznan, Poland

E-mail:kgo.ela@wp.pl

${ }^{*}$ Contributed equally

Key words: endometrial adenocarcinoma, gastrointestinal stromal tumor, Meckel's diverticulum, microarrays, KIT gene revealing the contribution of the interstitial cells of Cajal, the spindle cells of the gut wall (1-4). The majority of GISTs are located within the stomach $(50-70 \%)$ or the small intestine (20-30\%), with as few as $10 \%$ of the tumors developing in the rectum, and only 5\% developing in the large intestine, the retroperitoneal space and a variety of other locations (i.e., appendix and pancreas) $(5,6)$. These tumors are even less frequent within the mesentery, omentum and esophagus (7).

The age of onset for GIST patients is broad, but the tumors commonly occur at 50-60 years. Precise GIST diagnostics became possible only after the 1998 discovery of the c-kit proto-oncogene and cluster of differentiation (CD)117 protein overexpression in the tumor cells (8). The biological nature of these tumors, indispensably link the activity of v-kit Hardy-Zuckerman 4 feline sarcoma viral oncogene homolog (KIT) kinase and platelet-derived growth factor receptor $\alpha$ (PDGFRA) in cancer progression (9-11). Notably, $10-15 \%$ of tumor cases are not associated with these genes (designated as KIT/PDGFRA wild-type), but rather to different carcinogenesis contributors, such as the succinate dehydrogenase complex and mutations of neurofibromin 1, B-Raf proto-oncogene, serine/threonine kinase or Kirsten rat sarcoma viral oncogene homolog kinase $(12,13)$. This broad genetic heterogeneity of GIST highlights the complexity of the tumor origin, but more importantly, further affects the varied responsiveness of GISTs to treatment with the tyrosine kinase inhibitors (14).

Meckel's diverticulum (MD) is the most frequent congenital defect of the small intestine and is present in $\sim 2 \%$ of the general population. Neoplasms of MD are diagnosed only in 0.5-3.2\% of the population carrying this anatomical defect (15). To date, GISTs of MD origin have not been investigated thoroughly at the genomic level. However, a few case studies have described the tumor tissue examination, showing positive immunohistochemistry reactions for vimentin and c-kit, therefore indicating the GIST nature (15-17). A previous epidemiological study on 163 MD cases has indicated that MD is a cancer 'hot-spot', comprising an attractive location for tumor development (18). Moreover, when comparing different types of cancer, an apparent preponderance of adenocarcinomas versus malignant carcinoids (2:1 ratio) was observed. 
The present study attempted to comprehensively explore and reveal the genetic nature of the rare cancer tissue located in MD and investigate its GIST origin.

\section{Patients and methods}

Patient. A 71-year-old woman reported to the Obstetrics and Gynecology Emergency Room (Clinical Hospital, Poznan, Poland) on December 3, 2011, due to newly occurring postmenopausal bleeding. A biopsy of the endometrium was performed and the material obtained was assessed by the Pathology Laboratory. The results of the histopathological examination indicated endometrioid adenocarcinoma (G1). Following the diagnosis, the patient was admitted to the hospital for further treatment. Subsequent to being admitted to the Surgical Gynecology Clinic of the Gynecological and Obstetrics Clinical Hospital, the patient underwent a gynecological examination and a transvaginal ultrasound. Following a cardiology consultation, the patient qualified for elective surgical treatment. During the surgery, the entire uterus with adnexa was removed, and a solid tumor of $\sim 3 \mathrm{~cm}$ in size, which was previously not visible in the ultrasound image, was found in MD. The procedure included a complete resection of the tumor along with the diverticulum and an end to end intestinal anastomosis. Tissue samples were sent for a histopathological examination. No intraoperative examinations were performed. There were no complications in the post-operative period or during recovery following the intestinal anastomosis. The patient was discharged from the hospital on the 7th day after the surgery in a good general condition. The patient was advised to await the histopathological examination results and was informed that a decision concerning any further course of treatment would be made based on these results.

Genetic examination. Once the treatment was completed, advanced molecular testing was employed in order to identify tumor-specific genome changes. Briefly, the Genome-Wide Human CytoScan HD Array and CytoScan 750K (Affymetrix, Santa Clara, CA, USA) was used to analyze genomic alterations in the tumor sample. Genomic DNA was obtained from formalin-fixed paraffin-embedded (FFPE) sections using conventional processing (deparaffinization, enzymatic treatment and DNA extraction) (19). The $250 \mathrm{ng}$ of genomic DNA from the tumor was subjected to microarray examination according to the manufacturer's protocols as follows: i) Digestion with the restriction enzyme NspI; ii) adapter ligation, polymerase chain reaction (PCR) amplification and magnetic bead purification; iii) fragmentation and end-labeling with biotin; iv) washing and staining using a GeneChip ${ }^{\circledR}$ Fluidics Station 450; and v) scanning using an Affymetrix GeneChip Scanner 3000 7G (Affymetrix). Scanned data files were generated using Affymetrix GeneChip Command Console Software, version 1.2, and analyzed with Affymetrix Chromosome Analysis Suite v 2.0.0.195 (Affymetrix). To calculate the copy number of altered regions, the data were normalized to baseline reference intensities using NA 32.3 FFPE v.2 reference model (Affymetrix). The hidden Markov model available within the software package was used to determine the copy number states $(\mathrm{CN})$ and their breakpoints. Thresholds of $\log _{2}$ ratio $\geq 0.58$ and $\leq 1$ were used to categorize altered regions as $\mathrm{CN}$ variation (CNV) gains (amplifications) and copy number losses (deletions), respectively. To prevent the detection of false-positive CNVs arising due to microarray unspecific signals, only regions that involved at least 50 consecutive probes were considered in the analysis of gains or losses in this study. Amplifications and deletions were analyzed separately. To exclude aberrations representing common normal CNVs, all the identified CNVs were compared with those reported in the Database of Genomic Variants (http://projects.tcag.ca/variation/). To identify the genes involved in the CNVs further, the UCSC database (http://genome.ucsc.edu) and Ensemble (http://www.ensembl.org) were used. Gene annotation and gene overlap were determined using the human genome build 19 and NetAffx (http://www.affymetrix.com). In addition, the identified alterations were compared with COSMIC database (http:// cancer.sanger.ac.uk) (20) to look for overlap with up-to-date, known genomic cancer regions and single cancer genes. The algorithm for the detection of copy number aberrations in tumor cell mixtures (mosaicism and clonality) considers the comprehensive analysis of adjacent single copy deletions and gains segments. The algorithm is designed to be most accurate when the normal/expected $\mathrm{CN}$ is diploid and targets the detection of changes in regions of $\sim 5 \mathrm{Mb}$ or more in size and variation with a minimum of 500 markers (being typical for segments of 5,000 markers or more). This approach considers only a discrete number of mosaicism levels, which are set at 30,50 and $70 \%$. The range of log ratios is broken into a series of bands according to the detection level ( $\geq 30, \geq 50 \%$ or $70-100 \%$ bands) and log ratios within each band denote a specific copy number change event. This tool is most efficient in detecting mosaicism between $30-70 \%$ of cells and for copy numbers between 1 and 3. Selected regions were validated in the present study using quantitative PCR (2- $-\Delta \mathrm{Cq} ;$ KIT gene).

\section{Results}

Clinical findings. The results of the histopathological examination indicated endometrioid adenocarcinoma (G1) and a uterine leiomyoma; GIST, T2 (immunophenotype: CD117 ${ }^{+}, \mathrm{CD} 34^{+}$ and vimentin ${ }^{+}$). Laboratory tests, a gynecological examination and transvaginal ultrasound were performed during the next hospital stay. No indications for chemotherapy were identified, the radical nature of the surgery was confirmed, as well as the complete removal of the lesion. After a single day of in-patient treatment, the patient was discharged in a good general condition and was recommended to undergo whole-body positron emission tomography-computed tomography. The scan was performed 12 months after the last hospitalization. No pathological tracer uptake areas were identified. At present, the patient is under the care of the Oncology Outpatient Clinic. No episodes of recurrence had been identified at the time of preparing the manuscript for this case study.

Genomic studies. The strategy for the reliable detection of chromosomal rearrangements assumes usage of the CytoScan $750 \mathrm{~K}$ chip in the first step. This investigation resulted in detection of $\sim 62$ genomic imbalances that were not filtered out and passed the genomic criteria ( $>400 \mathrm{~kb} ; 50$ markers). To confirm abnormalities and fine mapping of the borders for genomic segments, a high-resolution chip Cytoscan HD (2.7 million probes and high coverage of 522 cancer genes) was applied. The 


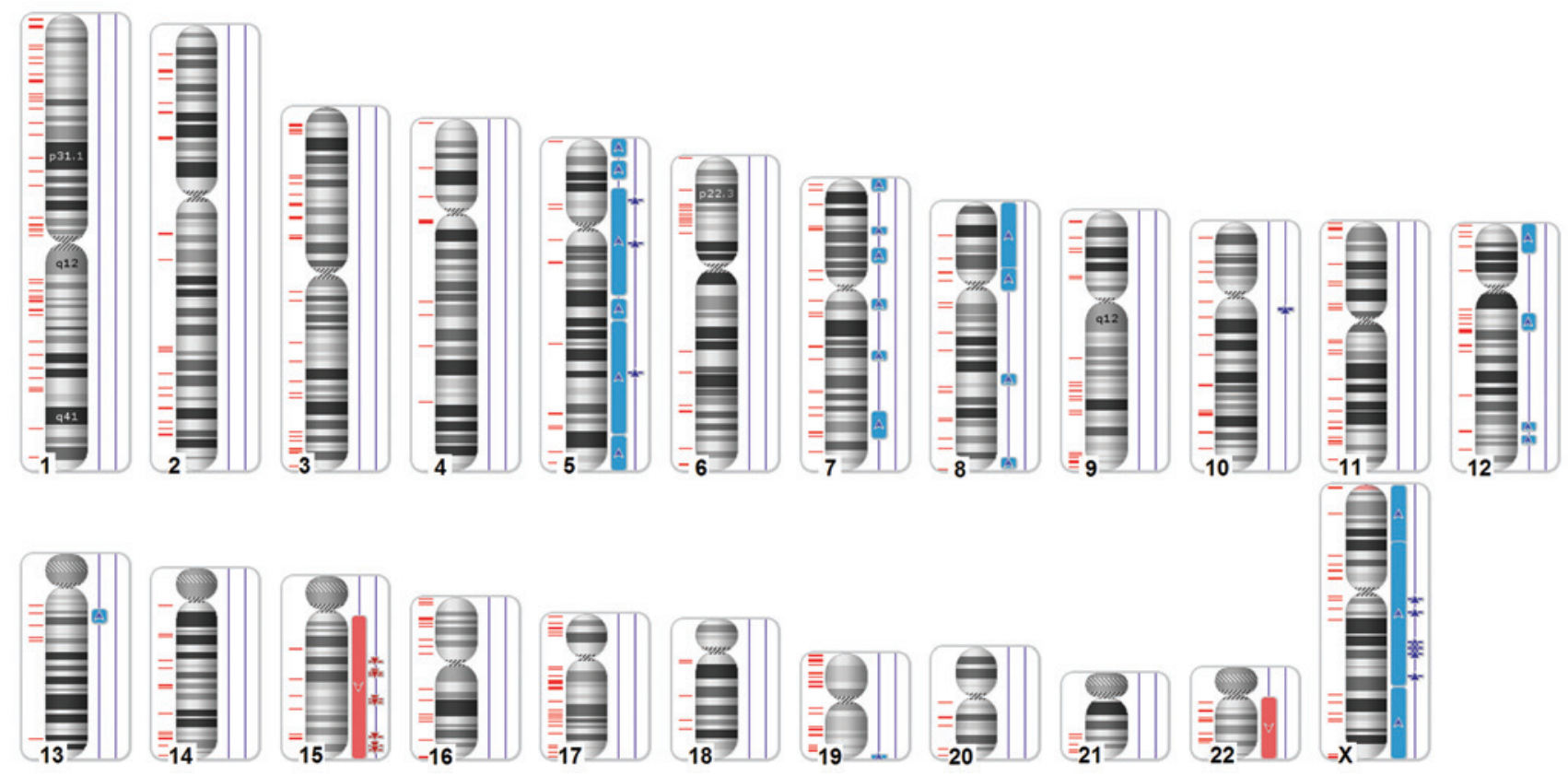

Figure 1. A karyoview of identified genomic abnormalities (right of each ideogram). Gains are shown in blue bars (regions of $\mathrm{CN} 2-3$ in light blue and $\mathrm{CN} \geq 3$ in dark blue). Losses are shown analogically in red bars (regions of CN1-2 in light red and $\mathrm{CN} \leq 1$ in dark red). The location and distribution of 522 cancer genes (COSMIC) are indicated on the left of ideogram. CN, copy number states.

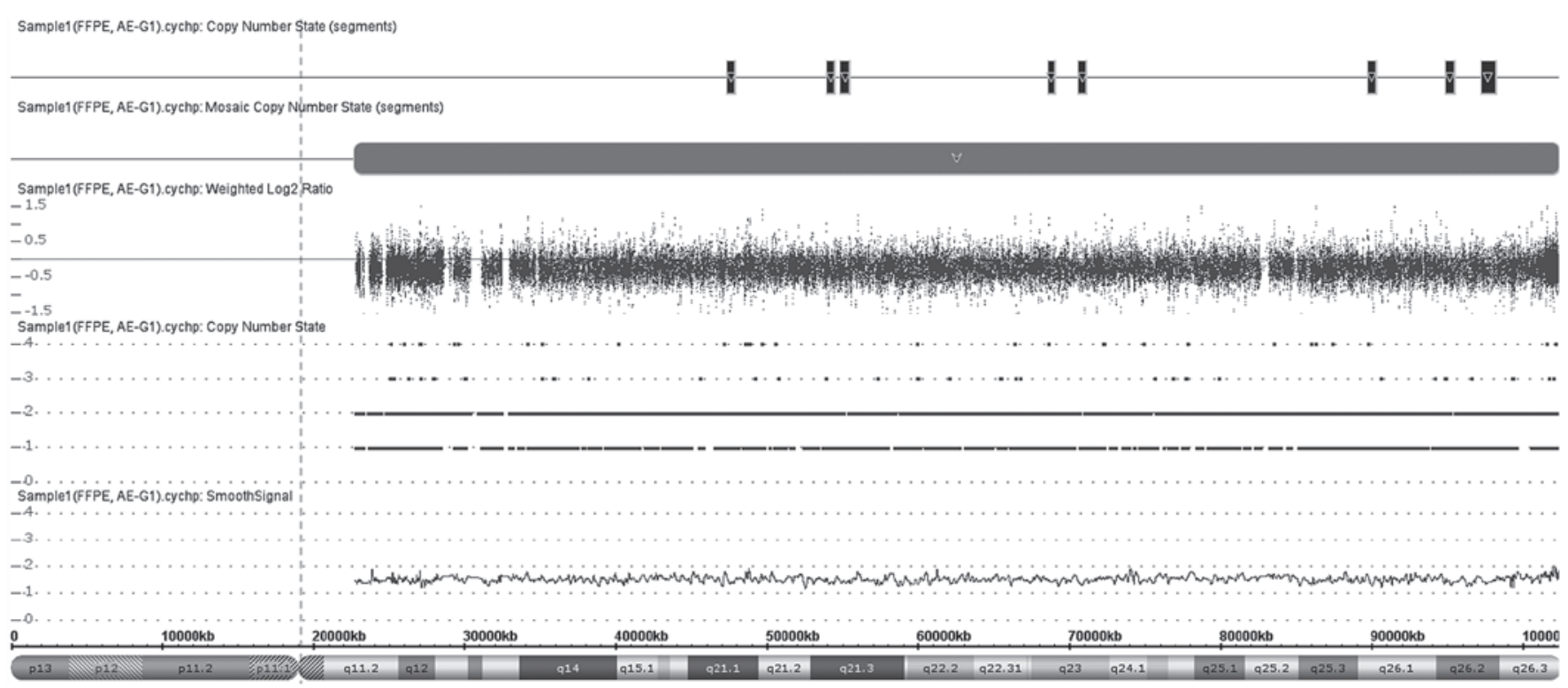

Figure 2. An example of microarray analysis of chromosome 15. $\log _{2}$ ratio and copy number state in examined formalin-fixed paraffin-embedded (FFPE) tumor tissue are shown.

unbiased density of probes on the chip enabled improvements to the accuracy and confidence of detected regions. A total of 45 previously detected regions were confirmed, and 17 other segments were disregarded as false-positives (signal noise, more rigorous filtering criteria compared with CytoScan $750 \mathrm{~K}$ chip and usage of FFPE reference model for Cytoscan HD that is far more accurate). In total, the cancer tissue revealed alterations on 6 chromosomes (chromosomes 7, 8, 10, 12, 13 and 19) and 4 entire chromosome aneuploidies (chromosomes 5, 15, 22 and $\mathrm{X}$ ). A dedicated karyogram illustrating the localization of each segment is shown in Fig. 1, and an example of mosaic aneuploidy (chromosome 15) is shown in Fig. 2. The genomic regions where than divided into 4 groups: Low mosaic gains (CN2-3), low mosaic losses (CN2-1), amplifications ( $\mathrm{CN}>3$ ) and deletions suggestive for loss of heterozygosity $(\mathrm{CN}<1)$. All these data and precise genomic coordinates were incorporated into Table I. Within detected intervals, 4,803 genes were identified. Having high microarray reproducibility, less rigorous criteria were applied to narrow down the abnormality size up to $100 \mathrm{~kb}$ (with 50 markers unchanged) to evaluate smaller rearrangements, expanding the genomic area to 442 segments. A focus was placed particularly on known cancer genes (522 entities) and 


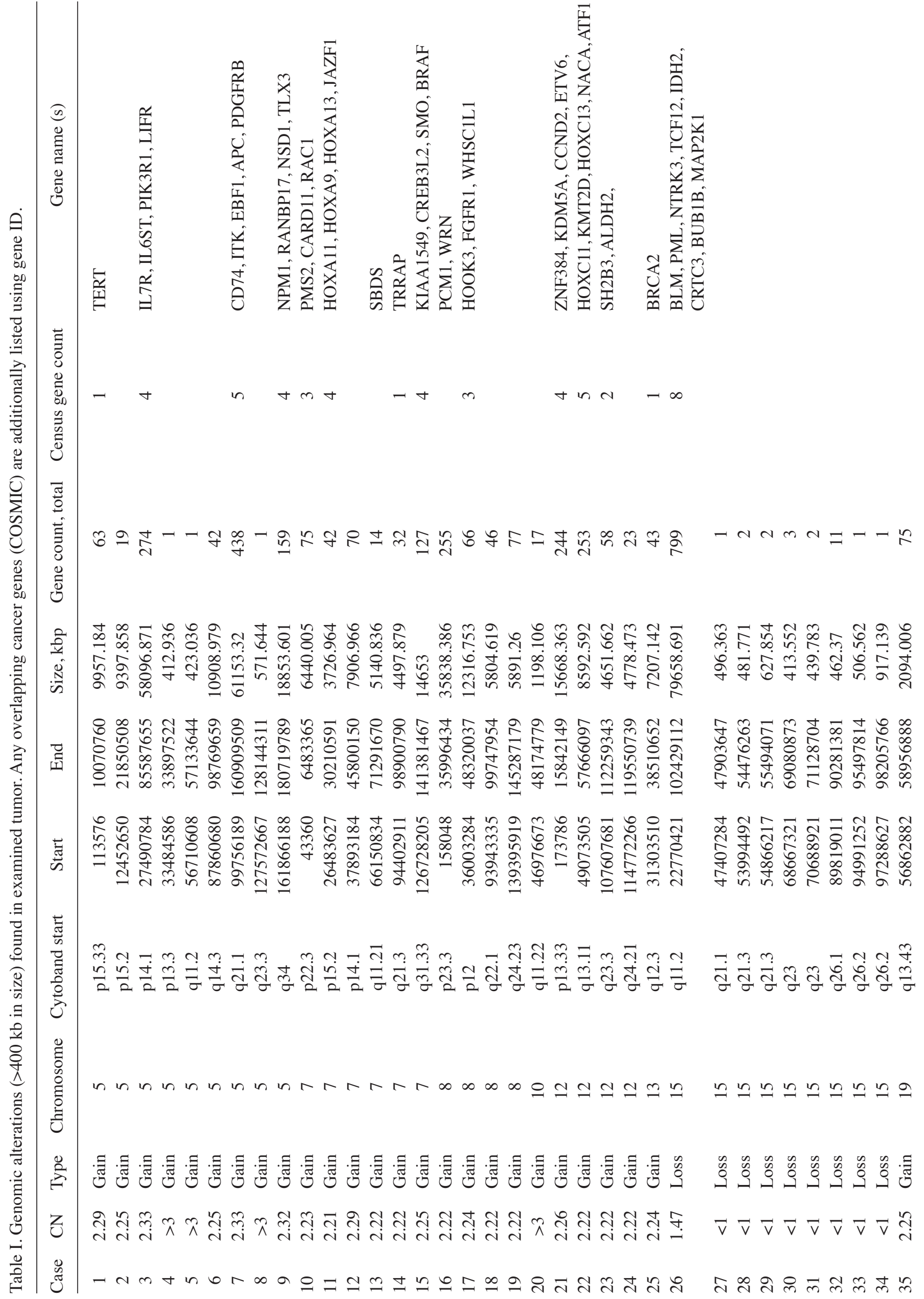




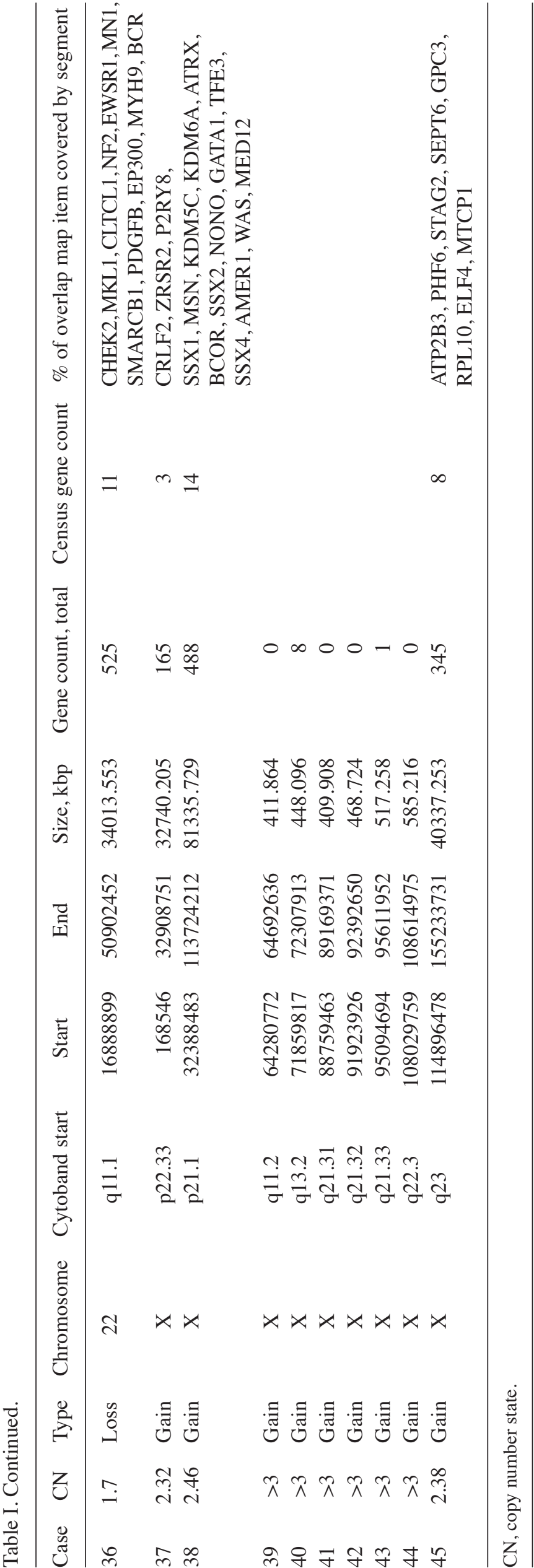

Table II. COSMIC database reporting genes to be mutated in gastrointestinal tract (site indeterminate and various tumor types) and small intestine adenocarcinoma. ${ }^{a}$

\begin{tabular}{|c|c|c|c|}
\hline Case & Gene & Mutated samples & Sample tested \\
\hline 1 & NRAS & 79 & 502 \\
\hline 2 & $H R A S$ & 31 & 495 \\
\hline 3 & TERT & 28 & 97 \\
\hline 4 & $K R A S$ & 19 & 490 \\
\hline 5 & $P I K 3 C A$ & 14 & 194 \\
\hline 6 & PTEN & 11 & 150 \\
\hline 7 & $B R A F$ & 8 & 595 \\
\hline 8 & $T S H R$ & 8 & 50 \\
\hline 9 & $I D H 1$ & 6 & 53 \\
\hline 10 & $C D K N 2 A$ & 4 & 28 \\
\hline 11 & $M E T$ & 2 & 41 \\
\hline 12 & TP53 & 2 & 20 \\
\hline 13 & GNAS & 2 & 73 \\
\hline 14 & PAX8 & 0 & 142 \\
\hline 15 & $E G F R$ & 0 & 101 \\
\hline 16 & CCDC6 & 0 & 80 \\
\hline 17 & PDGFRA & 0 & 64 \\
\hline 18 & KIT & $\mathbf{0}$ & 64 \\
\hline 19 & $N C O A$ & 0 & 55 \\
\hline 20 & GNA11 & 0 & 46 \\
\hline 21 & $G N A Q$ & 0 & 42 \\
\hline 22 & PIK3R1 & $\mathbf{0}$ & 34 \\
\hline 23 & IDH2 & $\mathbf{0}$ & 32 \\
\hline 24 & $A K T 1$ & 0 & 23 \\
\hline 25 & $A L K$ & 0 & 22 \\
\hline 26 & RET & 0 & 21 \\
\hline 27 & PRKARIA & 0 & 16 \\
\hline 28 & MEN1 & 0 & 15 \\
\hline 29 & $A P C$ & $\mathbf{0}$ & 15 \\
\hline 30 & MAP2K1 & $\mathbf{0}$ & 13 \\
\hline 31 & STRN & 0 & 11 \\
\hline 32 & $V H L$ & 0 & 10 \\
\hline 33 & NPM1 & $\mathbf{0}$ & 10 \\
\hline 34 & $\mathrm{CDH1}$ & 0 & 8 \\
\hline 35 & $T F G$ & 0 & 6 \\
\hline 36 & $T P R$ & 0 & 6 \\
\hline 37 & $J A K 2$ & 0 & 6 \\
\hline 38 & CTNNBI & 0 & 4 \\
\hline 39 & $S F 3 B 1$ & 0 & 4 \\
\hline 40 & KDM6A & $\mathbf{0}$ & 4 \\
\hline 41 & SMAD4 & 0 & 4 \\
\hline 42 & PTPN11 & 0 & 3 \\
\hline 43 & ТРМ3 & 0 & 2 \\
\hline
\end{tabular}

${ }^{\mathrm{a}} \mathrm{Genes}$ contributing to the case and found within altered regions are in bold.

associated pathways that contribute to adenocarcinoma in MD. In order to divulge putative genes contributing to MD adenocarcinoma, the regions for COSMIC genes (known also as Census 
Table III. Kyoto Encyclopedia of Genes and Genomes pathway and statistical analysis for involvement of selected cancer genes present in altered genomic regions and their contribution to different cancer types or cellular control.

\begin{tabular}{|c|c|c|c|c|c|}
\hline Case & Gene ontology ID & Term & P-value & No. of genes & Genes \\
\hline 1 & hsa05200 & Pathways in cancer & $1.29 \times 10^{-6}$ & 12 & $\begin{array}{l}B C R, A P C, S M O, P D G F R B, \\
P D G F B, E P 300, B R A F, \\
M A P 2 K 1, P I K 3 R 1, B R C A 2, \\
F G F R 1, P M L\end{array}$ \\
\hline 2 & hsa05215 & Prostate cancer & $1.27 \times 10^{-7}$ & 8 & $\begin{array}{l}\text { CREB3L2, PIK3R1, PDGFRB, } \\
\text { PDGFB, EP300, FGFR1, BRAF, } \\
\text { MAP } 2 K 1\end{array}$ \\
\hline 3 & hsa05218 & Melanoma & $7.8 \times 10^{-6}$ & 6 & $\begin{array}{l}P I K 3 R 1, P D G F R B, P D G F B \\
F G F R 1, B R A F, M A P 2 K 1\end{array}$ \\
\hline 4 & hsa05214 & Glioma & $6.4 \times 10^{-5}$ & 5 & $\begin{array}{l}\text { PIK3R1, PDGFRB, PDGFB, BRAF, } \\
\text { MAP } 2 K 1\end{array}$ \\
\hline 5 & hsa04630 & Jak-STAT signaling pathway & $8.73 \times 10^{-5}$ & 7 & $\begin{array}{l}P I K 3 R 1, I L 7 R, C C N D 2, C R L F 2 \\
I L 6 S T, E P 300, L I F R\end{array}$ \\
\hline 6 & hsa05211 & Renal cell carcinoma & $1.08 \times 10^{-4}$ & 5 & $\begin{array}{l}P I K 3 R 1, P D G F B, E P 300, B R A F, \\
M A P 2 K 1\end{array}$ \\
\hline 7 & hsa05213 & Endometrial cancer & $4.39 \times 10^{-4}$ & 4 & $A P C, P I K 3 R 1, B R A F, M A P 2 K 1$ \\
\hline 8 & hsa05221 & Acute myeloid leukemia & $5.87 \times 10^{-4}$ & 4 & $P I K 3 R, P M L, B R A F, M A P 2 K 1$ \\
\hline 9 & hsa05210 & Colorectal cancer & $6.73 \times 10^{-4}$ & 4 & $A P C, P I K 3 R 1, B R A F, M A P 2 K 1$ \\
\hline 10 & hsa05212 & Pancreatic cancer & $1.04 \times 10^{-3}$ & 4 & $P I K 3 R 1, B R C A 2, B R A F, M A P 2 K 1$ \\
\hline 11 & hsa05220 & Chronic myeloid leukemia & $1.38 \times 10^{-3}$ & 4 & PIK3R1, BRAF, MAP2K1, BCR \\
\hline 12 & hsa04110 & Cell cycle & $1.51 \times 10^{-3}$ & 5 & $\begin{array}{l}C C N D 2, C H E K 2, S T A G 2, E P 300, \\
B U B 1 B\end{array}$ \\
\hline 13 & hsa04510 & Focal adhesion & $2.43 \times 10^{-3}$ & 6 & $\begin{array}{l}P D G F R B, P D G F B, B R A F \\
M A P 2 K 1, P I K 3 R 1, C C N D 2\end{array}$ \\
\hline 14 & hsa05223 & Non-small cell lung cancer & $5.86 \times 10^{-3}$ & 3 & $P I K 3 R 1, B R A F, M A P 2 K 1$ \\
\hline 15 & hsa04062 & Chemokine signaling pathway & $8.99 \times 10^{-3}$ & 5 & $\begin{array}{l}P I K 3 R 1, W A S, B R A F, M A P 2 K 1, \\
I T K\end{array}$ \\
\hline 16 & hsa04060 & $\begin{array}{l}\text { Cytokine-cytokine receptor } \\
\text { interaction }\end{array}$ & $9.63 \times 10^{-3}$ & 6 & $\begin{array}{l}\text { CRLF2, IL6ST, PDGFRB, PDGFB, } \\
\text { LIFR, IL7R }\end{array}$ \\
\hline
\end{tabular}

JAK/STAT, Janus kinase/signal transducer and activator of transcription.

Genes) (21-23) were assessed and 88 entities were selected, 43 of which were recurrent (Table II). In order to further delineate the putative cancer pathways, gene-interactions were searched for using the Kyoto Encyclopedia of Genes and Genomes (KEGG) pathway database (http://www.genome.jp/kegg) (24). In total, $16 \mathrm{KEGG}$ pathways were selected that are associated with the development of different types of cancer or cellular regulation processes (Table III). Interacting genes were visualized as a network using String version 9.1 (http://string-db.org) (Fig. 3) (25).

\section{Discussion}

Current studies are aimed at estimating the incidence of GIST cases in the population. Swedish studies have shown that currently, the number of novel GIST cases amounts to 15-16 million/year (26). A tumor may develop in any section of the gastrointestinal tract, as well as intraperitoneally and in the retroperitoneal space. The current state of knowledge and statistical data indicate that when a radical surgical excision of the tumor is possible, the 5-year survival rate is $50-60 \%$ (27,28). At the same time, literature references indicate that $80 \%$ of patients who undergo surgery experience local recurrence within 2 years of the procedure, and that liver metastases additionally appear in $50 \%$ of the patients $(29,30)$. In the case discussed in the present study, the patient was treated surgically for reasons other than a GIST. This problem is widely discussed in the literature. Approximately $40 \%$ of women with small intestine GISTs undergo surgery due to genital tract tumors (GIST mimicking pelvic disorders). The genetic mapping of major molecular contributors, tyrosine kinases KIT and PDGFRA (ref. NM_000222 and NM_006206), reveal that they are located very close to each other on chromosome $4(31,32)$ and are found to be mutated in up to $90 \%$ of GIST cases. For each, a gain of function mutations results in constitutive activation of the oncogenes, but the clinical consequences may differ significantly $(33,34)$. The biological consequences related to abnormal hyperactivity of both proteins observed in cancer cells for each gene implies the occurrence of a variety of possible mechanisms, 


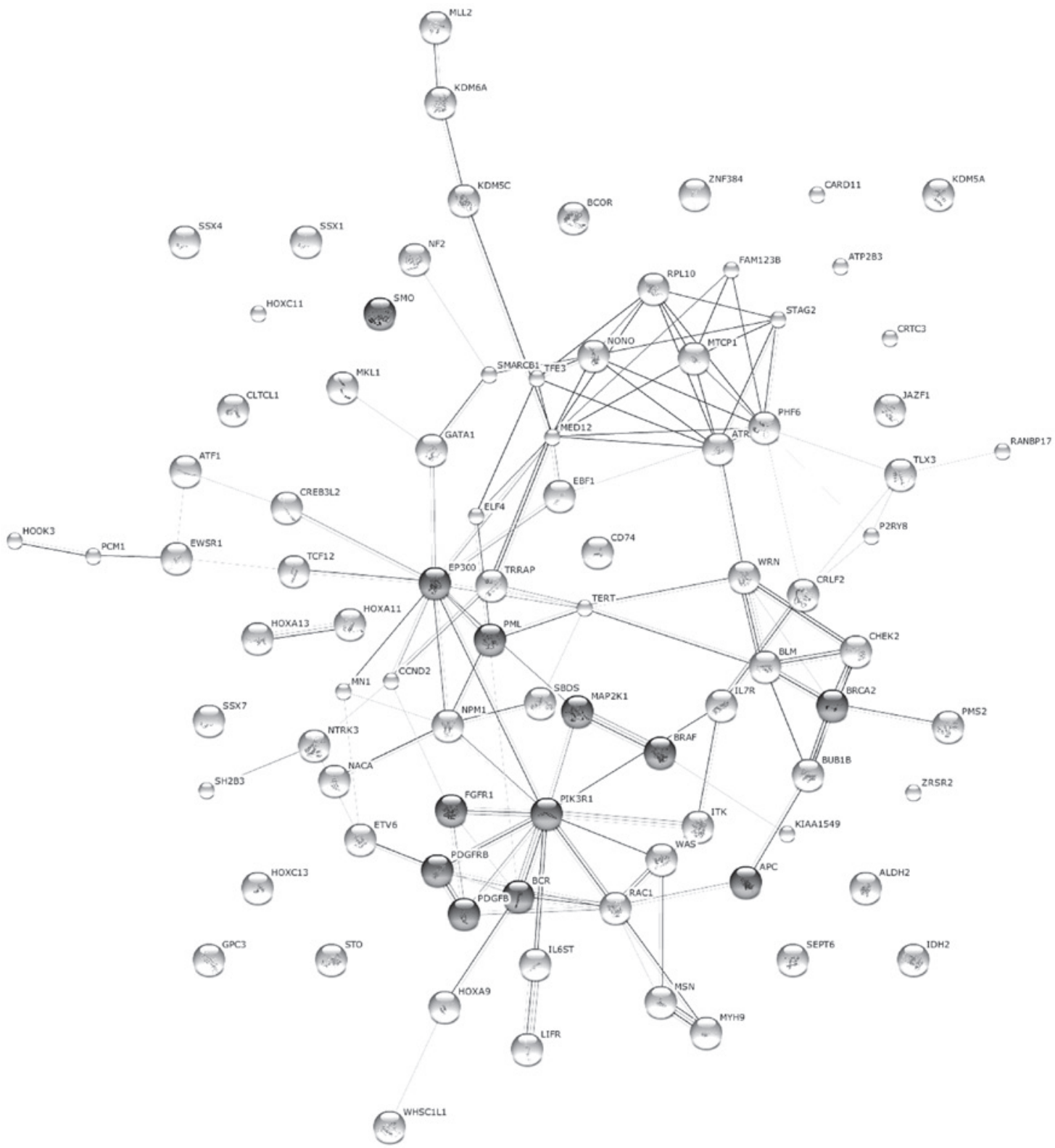

Figure 3. String visualization of the network interaction of all 87 selected cancer proteins. Proteins reported to be altered and contributing to Kyoto Encyclopedia of Genes and Genomes 'Pathways in cancer' (hsa05200; Table III) are indicated in dark gray (12 genes).

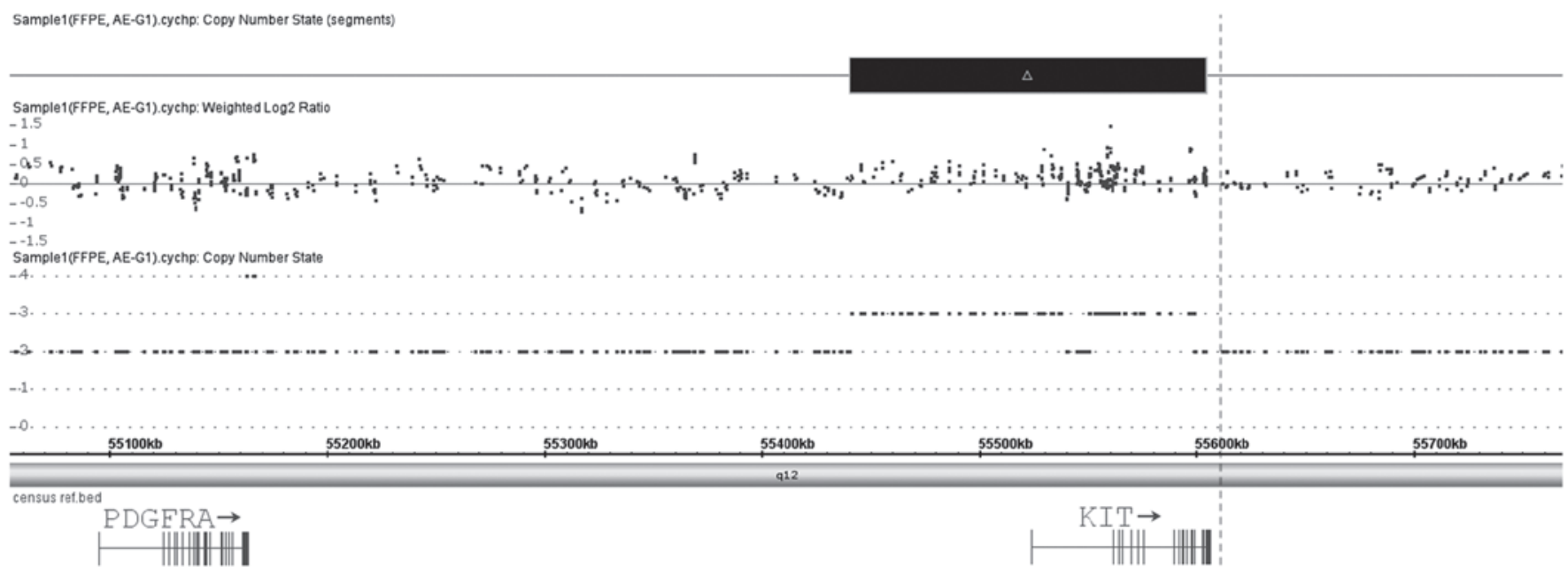

Figure 4. A high-resolution examination of $4 \mathrm{q} 12$ region presenting amplification of the KIT gene and no copy number state alteration of the PDGFRA gene. PDGFRA, platelet-derived growth factor receptor $\alpha$; FFPE, formalin-fixed paraffin-embedded. 
including point mutations and bigger genomic instabilities (whole gene amplifications or gene fusions). Hence, the comprehensive molecular portrait of GIST cancers is now emerging. This is possibly due to usage of high-throughput genomic approaches that provide valuable data referring to the involvement of a single gene, but also characterizing entire pathways on various stages of GIST carcinogenesis (35-37). To date, high-resolution mapping has been conducted only by two groups $(36,38)$, with reference to typical GIST occurrences. Specific chromosomal rearrangements are solid and a consistent finding accompanying KIT and PDGFRA alterations. Losses of chromosomes 1, 3, 13, 14, 15 and 22, whereas gains of chromosomes 4 and 5 may represent clinical utility and prognostic relevance. In the present case, a $15 q$ loss was identified, which is regarded as an aggressive course for a GIST. The loss of $1 p$ was also noted, which is typical for the small intestine localization of tumors, and the absence of a copy number alteration in the $R B 1$ locus, which is known as a strong clinical predictor (39). Application of a high-resolution microarray allows the identification of $\mathrm{CN}$ changes at a single gene level (for constitutional disorders, even single exon rearrangements). In the present study, KIT gene amplification was detected on chromosome 4, spanning only $163 \mathrm{~kb}$ and comprising the entire genomic sequence of this gene. Unexpectedly, the closely located PDGFRA gene (400 kb away) was not altered (Fig. 4). This finding is reminiscent and indicative of the principal role of KIT in triggering oncogenesis in this case. However, an activating point mutation in another gene cannot be excluded. Alteration of two prominent interacting proteins, PDGFRB and PIK3R1 (40), was also found. Phosphorylation of phosphatidylinositol 3-kinase (PIK3R1) by KIT leads to the activation of the v-akt murine thymoma viral oncogene homolog 1 signaling pathway. Activated KIT also transmits signals via growth factor receptor-bound protein 2 and activation of RAS, Raf-1 proto-oncogene, serine/threonine kinase and the mitogen-activated protein kinases (MAPKs) MAPK1/ERK2 and/or MAPK3/ERK1.

In conclusion, by employing a high-resolution microarray, the present study performed a comprehensive genomic analysis on genes contributing to GISTs, in the rare location of MD. A principal role of the KIT gene was confirmed in cancer initiation, which was demonstrated by detailed histopathological and molecular investigations. The detected chromosomal gains and losses were consistent with the findings of a GIST and confirm previous studies. Possible chemokine and cytokine-related signaling pathways (PIK3R1, BRAF, MAP2K1, PDGFRB and PDGFB) that may reasonably contribute to cancer progression with GIST characteristics were also indicated.

\section{Acknowledgements}

This study was supported by the grant no. 789/FNiTP/162/2013 Polish Ministry of Science and Education.

\section{References}

1. Sircar K, Hewlett BR, Huizinga JD, Chorneyko K, Berezin I and Riddell RH: Interstitial cells of Cajal as precursors of gastrointestinal stromal tumors. Am J Surg Pathol 23: 377-389, 1999.
2. Chan JK: Mesenchymal tumors of the gastrointestinal tract: A paradise for acronyms (STUMP, GIST, GANT, and now GIPACT), implication of c-kit in genesis, and yet another of the many emerging roles of the interstitial cell of Cajal in the pathogenesis of gastrointestinal diseases? Adv Anat Pathol 6: 19-40, 1999.

3. Rutkowski P, Debiec-Rychter M and Ruka W: Gastrointestinal stromal tumors: Key to diagnosis and choice of therapy. Mol Diagn Ther 12: 131-143, 2008.

4. Rutkowski P, Wozniak A, Dębiec-Rychter M, Kąkol M, Dziewirski W, Zdzienicki M, Ptaszynski K, Jurkowska M, Limon J and Siedlecki JA: Clinical utility of the new American Joint Committee on Cancer staging system for gastrointestinal stromal tumors: Current overall survival after primary tumor resection. Cancer 117: 4916-4924, 2011

5. Corless CL, Fletcher JA and Heinrich MC: Biology of gastrointestinal stromal tumors. J Clin Oncol 22: 3813-3825, 2004.

6. Miettinen M, Sarlomo-Rikala M and Lasota J: Gastrointestinal stromal tumors: Recent advances in understanding of their biology. Hum Pathol 30: 1213-1220, 1999.

7. Roberts PJ and Eisenberg B: Clinical presentation of gastrointestinal stromal tumors and treatment of operable disease. Eur J Cancer 38 (Suppl 5): S37-S38, 2002.

8. Neuhaus SJ, Clark MA, Hayes AJ, Thomas JM and Judson I: Surgery for gastrointestinal stromal tumour in the post-imatinib era. ANZ J Surg 75: 165-172, 2005.

9. Duensing A, Heinrich MC, Fletcher CD and Fletcher JA: Biology of gastrointestinal stromal tumors: KIT mutations and beyond. Cancer Invest 22: 106-116, 2004

10. Heinrich MC, Rubin BP, Longley BJ and Fletcher JA: Biology and genetic aspects of gastrointestinal stromal tumors: KIT activation and cytogenetic alterations. Hum Pathol 33: 484-495, 2002.

11. Huizinga JD, Thuneberg L, Klüppel M, Malysz J, Mikkelsen HB and Bernstein A: W/kit gene required for interstitial cells of Cajal and for intestinal pacemaker activity. Nature 373: 347-349, 1995.

12. Nannini M, Astolfi A, Urbini M, Indio V, Santini D, Heinrich MC, Corless CL, Ceccarelli C, Saponara M, Mandrioli A, et al: Integrated genomic study of quadruple-WT GIST (KIT/PDGFRA/SDH/RAS pathway wild-type GIST). BMC Cancer 14: 685, 2014.

13. Pantaleo MA, Nannini M, Corless CL and Heinrich MC: Quadruple wild-type (WT) GIST: Defining the subset of GIST that lacks abnormalities of KIT, PDGFRA, SDH, or RAS signaling pathways. Cancer Med 4: 101-103, 2014.

14. Heinrich MC, Griffith DJ, Druker BJ, Wait CL, Ott KA and Zigler AJ: Inhibition of c-kit receptor tyrosine kinase activity by STI 571, a selective tyrosine kinase inhibitor. Blood 96: 925-932, 2000.

15. Chandramohan K, Agarwal M, Gurjar G, Gatti RC, Patel MH, Trivedi P and Kothari KC: Gastrointestinal stromal tumour in Meckel's diverticulum. World J Surg Oncol 5: 50, 2007.

16. Khoury MG 2nd and Aulicino MR: Gastrointestinal stromal tumor (GIST) presenting in a Meckel's diverticulum. Abdom Imaging 32: 78-80, 2007.

17. Kosmidis C, Efthimiadis C, Levva S, Anthimidis G, Baka S,Grigoriou M,Tzeveleki I, Masmanidou M,Zaramboukas T and Basdanis G: Synchronous colorectal adenocarcinoma and gastrointestinal stromal tumor in Meckel's diverticulum; an unusual association. World J Surg Oncol 7: 33, 2009.

18. Thirunavukarasu P, Sathaiah M, Sukumar S, Bartels CJ, Zeh H III, Lee KK and Bartlett DL: Meckel's diverticulum - a high-risk region for malignancy in the ileum. Insights from a population-based epidemiological study and implications in surgical management. Ann Surg 253: 223-230, 2011.

19. Gillio-Tos A, De Marco L, Fiano V, Garcia-Bragado F, Dikshit R, Boffetta P and Merletti F: Efficient DNA extraction from 25-year-old paraffin-embedded tissues: Study of 365 samples. Pathology 39: 345-348, 2007.

20. Forbes SA, Bindal N, Bamford S, Cole C, Kok CY, Beare D, Jia M, Shepherd R, Leung K, Menzies A, et al: COSMIC: Mining complete cancer genomes in the Catalogue of Somatic Mutations in Cancer. Nucleic Acids Res 39 (Database): D945-D950, 2011.

21. Futreal PA, Coin L, Marshall M, Down T, Hubbard T, Wooster R, Rahman N and Stratton MR: A census of human cancer genes. Nat Rev Cancer 4: 177-183, 2004.

22. Pérez de Castro I, de Cárcer G and Malumbres M: A census of mitotic cancer genes: New insights into tumor cell biology and cancer therapy. Carcinogenesis 28: 899-912, 2007.

23. Santarius T, Shipley J, Brewer D, Stratton MR and Cooper CS: A census of amplified and overexpressed human cancer genes. Nat Rev Cancer 10: 59-64, 2010. 
24. Altermann E and Klaenhammer TR: PathwayVoyager: Pathway mapping using the Kyoto Encyclopedia of Genes and Genomes (KEGG) database. BMC Genomics 6: 60, 2005.

25. Franceschini A, Szklarczyk D, Frankild S, Kuhn M, Simonovic M, Roth A, Lin J, Minguez P, Bork P, von Mering C, et al: STRING v9.1: Protein-protein interaction networks, with increased coverage and integration. Nucleic Acids Res 41 (D1): D808-D815, 2013 .

26. Nilsson B, Bümming P, Meis-Kindblom JM, Odén A, Dortok A, Gustavsson B, Sablinska K and Kindblom LG: Gastrointestinal stromal tumors: The incidence, prevalence, clinical course, and prognostication in the preimatinib mesylate era - a population-based study in western Sweden. Cancer 103: 821-829, 2005.

27. Dematteo RP, Heinrich MC, El-Rifai WM and Demetri G: Clinical management of gastrointestinal stromal tumors: Before and after STI-571. Hum Pathol 33: 466-477, 2002.

28. Tosoni A, Nicolardi L and Brandes AA: Current clinical management of gastrointestinal stromal tumors. Expert Rev Anticancer Ther 4: 595-605, 2004

29. Ruka W, Rutkowski P, Nowecki Z, Nasierowska-Guttmejer A and Debiec-Rychter M: Other malignant neoplasms in patients with gastrointestinal stromal tumors (GIST). Med Sci Monit 10 LE13-LE14, 2004.

30. Rutkowski P, Nowecki ZI, Michej W, Debiec-Rychter M, Woźniak A, Limon J, Siedlecki J, Grzesiakowska U, Kakol M, Osuch C, et al: Risk criteria and prognostic factors for predicting recurrences after resection of primary gastrointestinal stromal tumor. Ann Surg Oncol 14: 2018-2027, 2007.

31. Hirota S, Isozaki K, Moriyama Y, Hashimoto K, Nishida T, Ishiguro S, Kawano K, Hanada M, Kurata A, Takeda M, et al: Gain-of-function mutations of c-kit in human gastrointestinal stromal tumors. Science 279: 577-580, 1998.

32. Chompret A, Kannengiesser C, Barrois M, Terrier P, Dahan P, Tursz T, Lenoir GM and Bressac-De Paillerets B: PDGFRA germline mutation in a family with multiple cases of gastrointestinal stromal tumor. Gastroenterology 126: 318-321, 2004.
33. Kwon JE, Kang HJ, Kim SH, Lee YC, Hyung WJ, Noh SH, Kim NK and Kim H: Pathological characteristics of gastrointestinal stromal tumours with PDGFRA mutations. Pathology 41: 544-554, 2009.

34. Schaefer IM, Ströbel P,Cameron S, Beham A, Otto C, Schildhaus HU and Agaimy A: Rhabdoid morphology in gastrointestinal stromal tumours (GISTs) is associated with PDGFRA mutations but does not imply aggressive behaviour. Histopathology 64: 421-430, 2014.

35. Gunawan B, Bergmann F, Höer J, Langer C, Schumpelick V, Becker H and Füzesi L: Biological and clinical significance of cytogenetic abnormalities in low-risk and high-risk gastrointestinal stromal tumors. Hum Pathol 33: 316-321, 2002.

36. Schoppmann SF, Vinatzer U, Popitsch N, Mittlböck M, Liebmann-Reindl S, Jomrich G, Streubel B and Birner P: Novel clinically relevant genes in gastrointestinal stromal tumors identified by exome sequencing. Clin Cancer Res 19: 5329-5339, 2013.

37. Wozniak A, Sciot R, Guillou L, Pauwels P, Wasag B, Stul M, Vermeesch JR, Vandenberghe P, Limon J and Debiec-Rychter M: Array CGH analysis in primary gastrointestinal stromal tumors: Cytogenetic profile correlates with anatomic site and tumor aggressiveness, irrespective of mutational status. Genes Chromosomes Cancer 46: 261-276, 2007.

38. Astolfi A, Nannini M, Pantaleo MA, Di Battista M, Heinrich MC Santini D, Catena F, Corless CL, Maleddu A, Saponara M, et al: A molecular portrait of gastrointestinal stromal tumors: An integrative analysis of gene expression profiling and high-resolution genomic copy number. Lab Invest 90: 1285-1294, 2010.

39. Lagarde P, Pérot G, Kauffmann A, Brulard C, Dapremont V, Hostein I, Neuville A, Wozniak A, Sciot R, Schöffski P, et al: Mitotic checkpoints and chromosome instability are strong predictors of clinical outcome in gastrointestinal stromal tumors. Clin Cancer Res 18: 826-838, 2012.

40. Liu CH, Chen TC, Chau GY, Jan YH, Chen CH, Hsu CN, Lin KT, Juang YL, Lu PJ, Cheng HC, et al: Analysis of protein-protein interactions in cross-talk pathways reveals CRKL protein as a novel prognostic marker in hepatocellular carcinoma. Mol Cell Proteomics 12: 1335-1349, 2013. 